\title{
Rogue waves in a wave tank: experiments and modeling
}

\author{
A. Lechuga \\ CEDEX, Ministry of Fomento, Madrid, Spain \\ Correspondence to: A. Lechuga (antonio.lechuga@cedex.es)
}

Received: 23 May 2013 - Published in Nat. Hazards Earth Syst. Sci. Discuss.: 11 July 2013

Revised: 18 September 2013 - Accepted: 23 October 2013 - Published: 20 November 2013

\begin{abstract}
In past decades theoretical studies have been carried out with the double aim of improving knowledge of the main characteristics of the rogue wave and of attempting to predict its sudden appearance. We have tried to generate rogue waves in a water wave tank, using a symmetric spectrum (Akhmediev et al., 2011a) as input on the wave maker.

The next step has been to apply a theoretical model to the amplitude envelope of these waves. After some considerations we agreed the best model to be an analog of the Ginzburg-Landau equation.
\end{abstract}

\section{Introduction}

Recently, many rogue waves have been reported to be the main cause of shipping incidents at sea. One of the main characteristics of rogue waves is their elusiveness: they appear unexpectedly and disappear in the same way. Some authors (Zakharov et al., 2010) are attempting to discover the probability of their appearances as well as studying the mechanism of their formation. Similarly, more recently, some researchers (Bitner-Gregersen and Toffoli, 2012) have studied the probability of occurrence of rogue waves.

Generated waves were clearly rogue waves with a ratio (maximum wave height / significant wave height) of 2.33 and a kurtosis of 4.77 (Janssen, 2003 and Onorato et al., 2005). These results were already presented (Lechuga, 2012). Similar waves (in the pattern aspect, but without being extreme waves) were described as crossing waves in a water tank (Shemer and Kit, 1988). Other researchers (Pelinovsky et al., 2005) have studied the relationship between the experiment data and mechanisms of generation of rogue waves. Toffoli et al. (2011) have also studied crossing seas as a mechanism of generation of rogue waves. In order to model the resulting waves, we use an analog of the Ginzburg-Landau equation.
We know that the Ginzburg-Landau model is related to some regular structures on the surface of a liquid and also in plasmas, electric and magnetic fields, and other media. Another important aspect of the model is that the solutions are invariants with respect to the translation group.

The main aim of this paper is to draw conclusions from the model and make comparisons with the measured waves in the water tank. The nonlinear structure of waves and their regularity make the use of the Ginzburg-Landau model suitable for the amplitude envelope of generated waves in the tank, thus giving us a powerful tool to compare with the results of our experiment.

\section{Experiment}

In order to reproduce waves in maritime reality, we normally use a Jonswap spectrum. However, when we use a more symmetric spectrum, either in shallow or in deep water, the energy concentrates itself and something similar to rogue waves happens. Controlling wave maker parameters, we can generate this kind of breather; its description is the main aim of this paper. A picture of our facility appears in Fig. 1, with the wave maker to the right. The wave tank has the following dimensions: $36.0 \mathrm{~m}$ long, $6.5 \mathrm{~m}$ wide and $1.5 \mathrm{~m}$ deep. In this tank we built a semi-submerged structure with a length of $10.5 \mathrm{~m}$ simulating a dike of $409.5 \mathrm{~m}$. The wave maker is the piston-flap type with a rank of $0.80 \mathrm{~m}$, an MTS control system and NRC (Canada) GEDAP application with active absorption of reflexions. There are three wave sensors, one close to the wave maker, another below the footbridge that appears in Fig. 1, and a third close to the submerged structure, also shown in Fig. 1. Only the wave sensor close to the wave maker has slight differences with the measurement of the others. The shape of the waves is shown in Fig. 5. The waveform is preserved throughout the tank except for the 


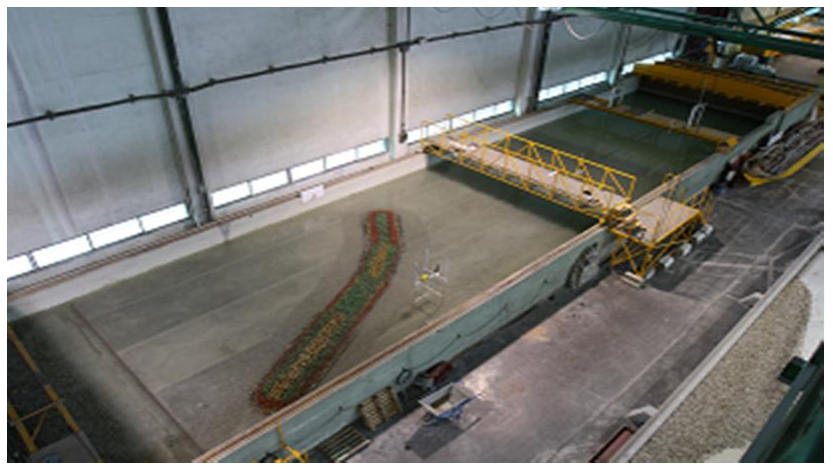

Fig. 1. Water wave tank.

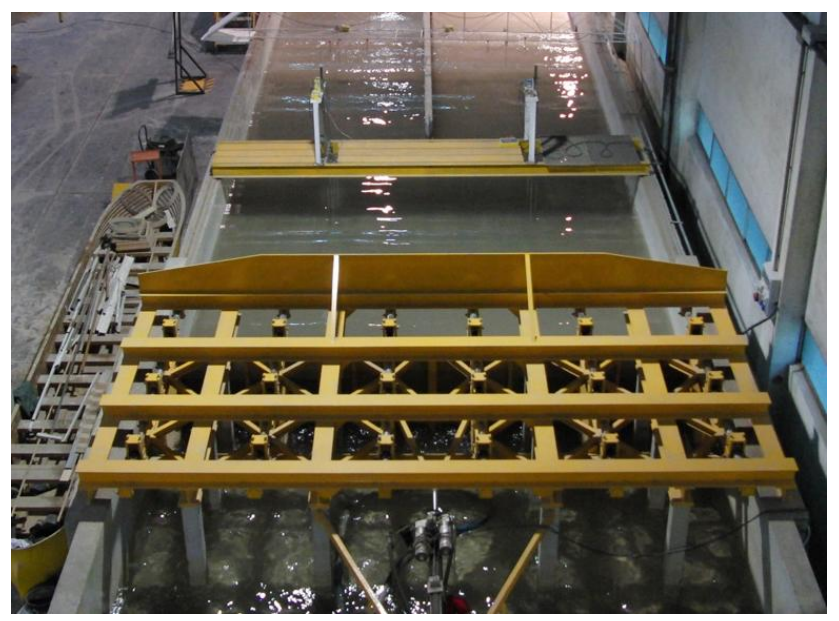

Fig. 2. Details of the wave maker with the double structure to prevent the appearance of vibrations.

perturbation produced in crossing the structure (see Fig. 5). The basic wave group is formed by three waves whose characteristics are shown in Table 1. We must take into consideration that our experiment, related to our study here, belongs to the deep water range.

As the main objective of our job was the generation of rogue waves, we will not dwell on other aspects related to the structure itself; however, we will point out that this dike has had to be built in Aviles Harbour in the north of Spain (Cantabrian Sea). On the right (see Fig. 1) is the wave maker. To eliminate transversal vibrations and so reproduce waves more accurately, the wave maker has a double structure as shown in Fig. 2.

We were trying different wave conditions, among them some waves generated with a density spectrum that has a strong symmetry (see the graphic in Fig. 3) and whose characteristics are shown in Figs. 4 and 5. The main aim of this truncated bimodal spectrum (following Akhmediev et al., 2011a) was to generate well-separated big waves with 5 or 6 small waves between them. There was an evident energy concentration similar to the one observed in photonic crys-

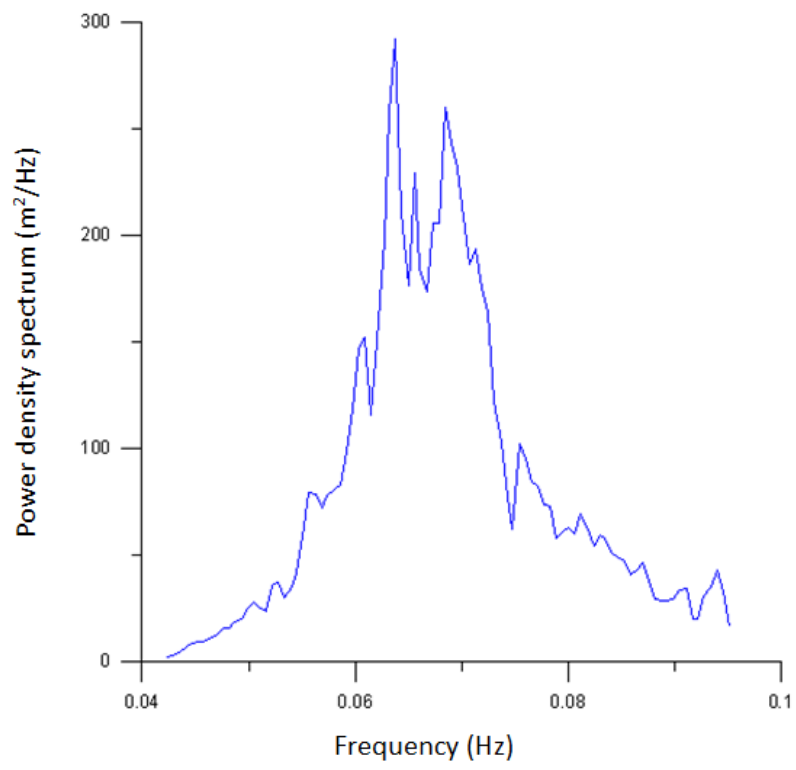

Fig. 3. Power density spectrum.

tal fiber in optical experiments. In our case the wave front is at right angles to the wave propagation, and accordingly Fig. 4 represents a wave profile. The observable energy concentration indicates that they can be considered rogue waves, at least from the statistical point of view. For instance, the ratio maximum height to significant height is between 2 and 2.33, and the kurtosis is between 4.77 and 9.83, far from the value of 3 of the Gaussian seas. Of course they are deterministic waves and therefore outside of the modulation instability mechanism and, also, they are almost symmetrical. For the same reasons the statistical values are included only for comparison purposes. Nevertheless their shape is similar to some waves reported as rogue waves (Fig. 6).

Zero up-crossing wave height has these values before and after the maximum wave (Table 1).

Table 1 represents the same results of Fig. 6, but computing the wave height of the three more relevant waves. Figure 6 shows a kind of zoom of the result of one of the wave sensors in time.

It is to be pointed out that the group so generated shows a conspicuous "three sister system".

\section{Theoretical background}

The Ginzburg-Landau equation has proved very useful for modeling the generated waves because it appears related to some regular structures on the surface of a liquid, as is the case in this experiment.

Following Danilov, Maslov and Volosov (1988), a special type of the Ginzburg-Landau equation can be represented as 


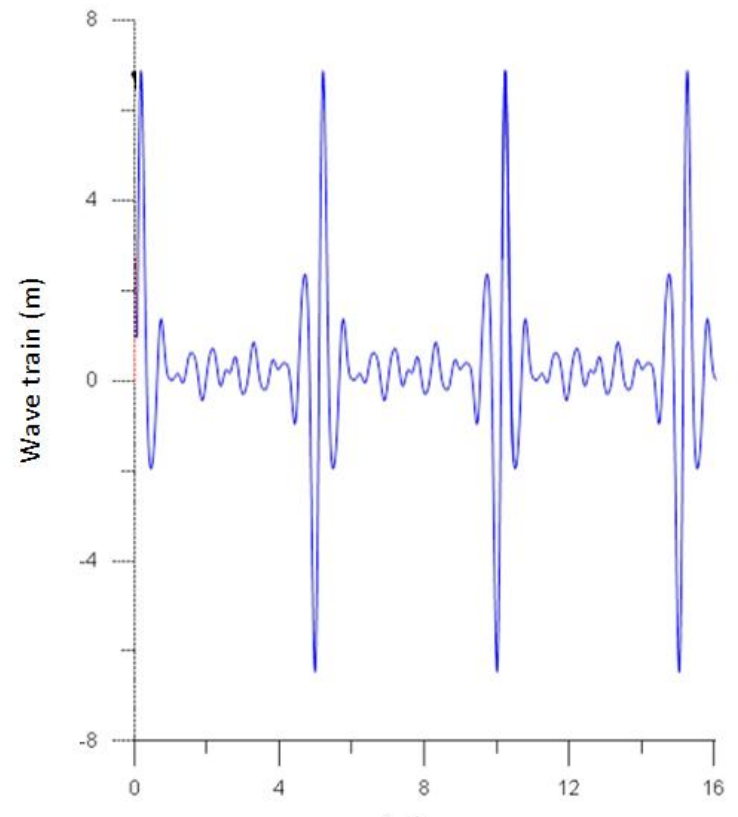

(m)

Fig. 4. Wave train profile. Axes have different units. For caption see Fig. 8.

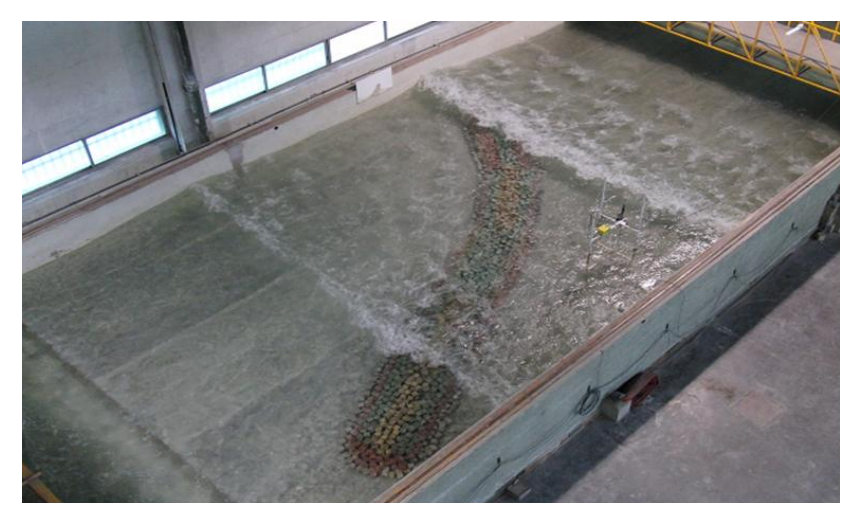

Fig. 5. Generated waves. Two big waves and small waves between them.

Table 1. Free wave surface close to the maximum wave. A group of 3 waves is noticeable.

\begin{tabular}{ccc}
\hline Number & $\begin{array}{c}\text { Wave Height } \\
\text { (meters) }\end{array}$ & Comments \\
\hline 1 & 1.1427 & \\
2 & 0.6610 & \\
3 & 3.2961 & \\
4 & 13.2299 & Maximum height \\
5 & 3.2958 & \\
\hline
\end{tabular}

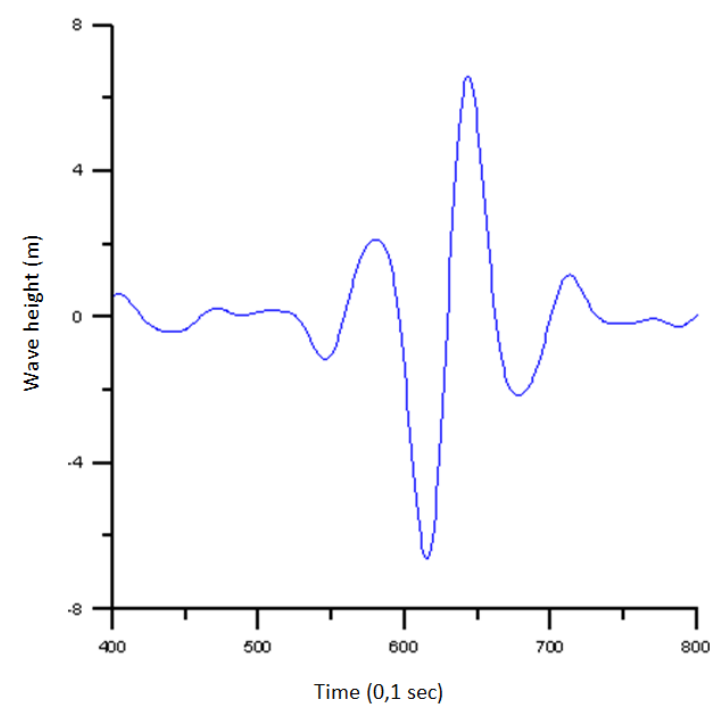

Fig. 6. Maximum wave height. One big wave accompanied by two smaller waves.

$\frac{\delta u}{\delta t}-i \sigma \varepsilon^{2} \frac{\delta^{2} u}{\delta x^{2}}+u-i h u \cdot-i u|u|^{2}+i \sigma u=0$

$u=\sqrt{\frac{\sigma}{2}}(U+i U)$

$U=U_{1}\left(\frac{x}{\varepsilon} \sqrt{\sigma}\right)$

$U_{1}=-\frac{\sqrt{2}}{\cosh \left(\frac{x \sqrt{\sigma}}{\varepsilon}\right)}$.

Though the steady solution only depends on $x$, it is necessary to use the complete GL Eq. (1) because we have to account for the real and imaginary parts of the solution.

As the solution of the equation is invariant to the translation group, we can repeat the number of separated waves as much as to reproduce our number of crests with our specific wave length.

$u=\sqrt{\frac{\sigma}{2}}(U+i U)$

$U=\sum_{l=1}^{3} U_{1}\left(\frac{x+l L}{\varepsilon} \sqrt{\sigma}\right)$,

where $L$ is the wavelength. Eq. (1) is dimensionless, and taking into account that it was not derived by water waves, the parameters have to be thought out for the new situation. 


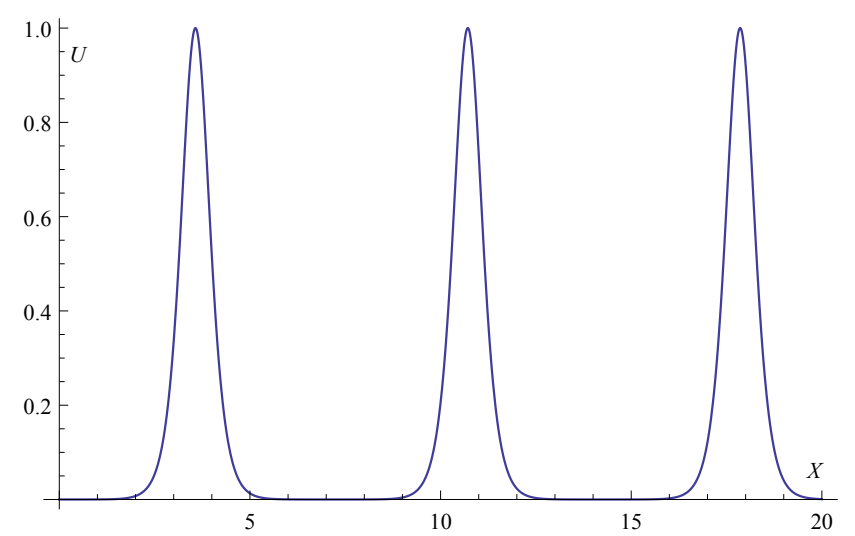

Fig. 7. Solution of the Ginzburg-Landau equation.

For instance, the solution for three well-separated waves is like this (see Fig. 7). In the plotted solution the ordinate and abscissa are dimensionless. The parameters that govern the equation are $\sigma$ and $\varepsilon: \sigma$ is involved in the amplitude of $u$ (real part) and in its "peakedness", and $\varepsilon$ in the wave peakedness. In Fig. 7, the parameter values are $\sigma=1$ and $\varepsilon=0.5$ ("peakedness" here does not have the precise meaning that it would in technical literature).

It is important to point out that with the two parameters we can adjust the solution of the Ginzburg-Landau equation to our generated waves. The steady solution of the GL equation only depends of the variable $x$, but you have to start with Eq. (1) to account for the real and imaginary parts that together provide the solution.

\section{Wave envelope}

In order to compare our experiment data with the solution of the Ginzburg-Landau equation, we proceed to find the amplitude envelope of our wave train, and to perform this we use the Hilbert transform (see Fig. 8).

This envelope (in profile) is similar to the Akhmediev breathers generated in optical fiber (Akhmediev et al., 2011b). The energy concentration in a few waves as well as the vanishing of intermediate waves between them is remarkable. The difference with the Akhmediev breathers is that in our case the system is bidimensional (plane crests at right angles to the wave propagation), whereas the former is clearly tridimensonal. However, the comparison of both profiles is relevant.

The structure of the amplitude envelope is easily modeled by the Ginzburg-Landau equation presented above, and there is no better model for this controlled experiment. The reason for that is to be found in two characteristics of the equation, i.e., its nonlinear structure and the regularity of the solutions. In our case the value of the parameters $\sigma, \varepsilon$ and $L$ are $\sigma=0.029, \varepsilon=0.085$ and $L=4.8$. Taking these values, the solution of the GL equation fits reasonably well with the

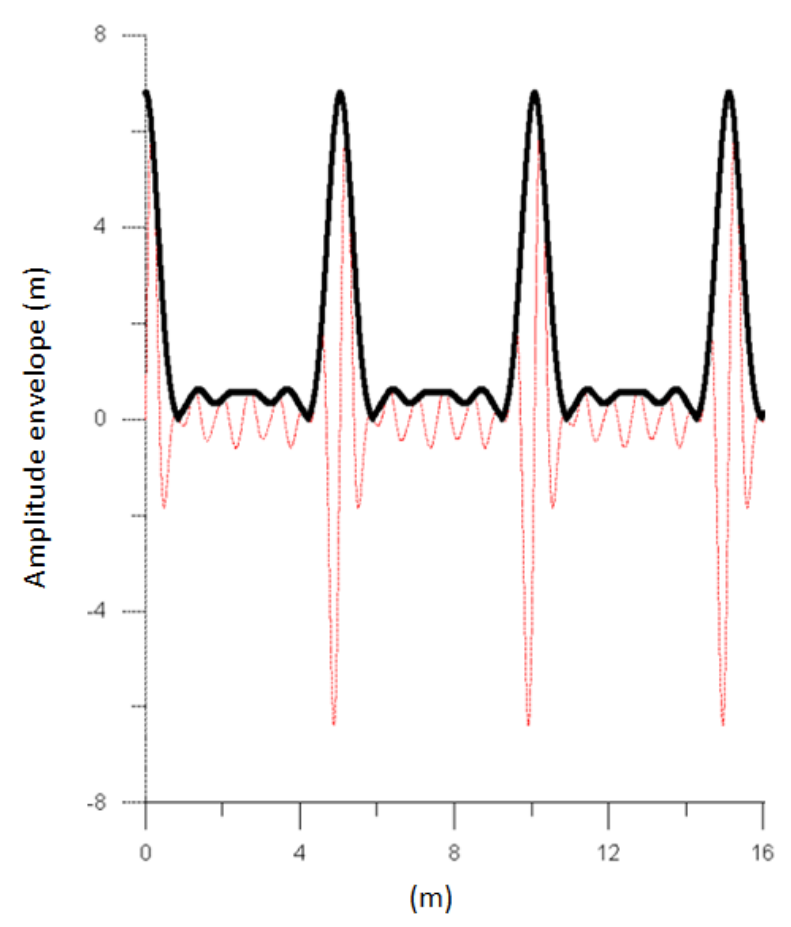

Fig. 8. Amplitude envelope (in black) of the wave train. The horizontal axis (abscissa) is in units of our wave tank. The ordinate units are in the scale of the model (1:39).

envelope of the generated waves. Alternatively we can consider the steady solution as dimensional, with $\sigma$ and $\varepsilon$ both in squared meters and $L$ in meters.

\section{Conclusions}

In some cases, a symmetric density spectrum could be a very powerful tool to use to generate extreme waves or rogue waves to check maritime structures. Though the procedure is a deterministic one, we can use it to get a greater concentration of the energy with higher and more separated waves for a given significant wave height.

The nonlinear structure of such waves and their regularity make the use of the Ginzburg-Landau equation suitable for modeling the amplitude envelope of the generated waves, giving us another way of controlling the results of our experiment.

Acknowledgements. The author appreciates the comments and suggestions of the three anonymous reviewers who have contributed to the improvement of the text. He also wishes to thank the editor E. Bitner-Gregersen.

Edited by: E. Bitner-Gregersen

Reviewed by: three anonymous referees 


\section{References}

Akhmediev, N., Ankiewicz, A., Soto-Crespo, J. M., and Dudley, J. M.: Universal triangular spectra in parametrically-driven systems, Phys. Lett. A, 375, 775-779, 2011a.

Akhmediev, N., Soto-Crespo, J. M., Ankiewicz, N., and Devine, A.: Early detection of rogue waves in a chaotic wave field, Phys. Lett. A, 375, 2999-3001, 2011b.

Bitner-Gregersen, E. M. and Toffoli, A.: On the probability of occurrence of rogue waves, Nat. Hazards Earth Syst. Sci., 12, 751762, doi:10.5194/nhess-12-751-2012, 2012.

Danilov, V. G., Maslov, V. P., and Volosov, K. A.: Mathematical Models in Computer-component Technology:Asymptotic Methods of Solution, in: mathematical Aspects of Computer Engineering, Mir Publisher, Moscow, 355-358, 1988.

Janssen, P. A.: Nonlinear four-wave interactions and freak waves, J. Phys. Oceanogr., 33, 863-884, 2003.

Lechuga, A.: Generation of rogue waves in a wave tank, Geophys. Res., 14,p. 1, 2012.
Onorato, M., Osborne, A., and Serio, M.: On deviation from Gaussian statistics for surface gravity waves, Proceedings Rogue Waves, Hawaiian Winter Workshop, 79-83,2005.

Pelinovsky, E., Kharif, C., Slunyaev, A., Talipova, T., and Sergeeva, A.: Freak waves: Physical mechanisms and experimental data, in: Proc Int. Conf., Frontiers of Nonlinear Physics, Nizhny Novgorov (Russia), 169-178, 2005.

Shemer, L. and Kit, E.: Study of the role of dissipation in evolution of nonlinear sloshing waves in a rectangular channel, Fluid Dyn. Res., 4, 89-105, 1988.

Toffoli, A., Bitner-Gregersen, E. M., Osborne, A. R., Serio, M., Monbaliu, J., and Onorato, M., Extreme waves in random crossing seas, Laboratory experiments and numerical simulations, Geophys. Res. Lett., 38, 1-5, 2011.

Zakharov, V. E., Dyachenko, A. I., and Shamin, R. V.: How probability for freak wave formation can be found, Eur. Phys. J. Spec. Top., 185, 113-124, 2010. 